\title{
Neutral shielding and cloaking of magnetic fields using isotropic media
}

Lars Kroon and Kenneth J ärrendahl

The self-archived postprint version of this journal article is available at Linköping University Institutional Repository (DiVA):

http:/ / urn.kb.se/ resolve?urn=urn:nbn:se:liu:diva- 145069

N.B.: When citing this work, cite the original publication.

Kroon, L., Järrendahl, K., (2017), Neutral shielding and cloaking of magnetic fields using isotropic media, J ournal of Physics, 29(3), . https:// doi.org/ 10.1088/ 0953-8984/ 29/3/ 035801

Original publication available at:

https:/ / doi.org/ 10.1088/ 0953-8984/29/3/035801

Copyright: IOP Publishing (Hybrid Open Access)

http:// www.iop.org/ 


\title{
Neutral shielding and cloaking of magnetic fields using isotropic media
}

\author{
Lars Kroon and Kenneth Järrendahl \\ Department of Physics, Chemistry and Biology, Linköping University, SE-581 83 \\ Linköping, Sweden \\ E-mail: lars.kroon@liu.se
}

\begin{abstract}
A method for designing magnetic shields that do not perturb applied multipole fields in the static regime is developed. Cylindrical core-shell structures with two layers characterized by homogeneous isotropic permeabilities are found to support neutral shielding of multipole fields and unique cloaking solutions of arbitrary multipole order. An extra degree of freedom is provided by every layer added to the structure which may be exploited with an effective design formula for cloaking of additional field terms. The theory is illustrated with numerical simulations
\end{abstract}

Keywords: magnetic shielding, neutral inclusion, cloaking, multipole fields

Submitted to: J. Phys.: Condens. Matter 


\section{Introduction}

Electromagnetic shielding is the practice of reducing the electromagnetic field in a space with barriers made of conductive or magnetic materials. Passive shields can reduce the electromagnetic interference between devices and their environments, and they are necessary for the reliable operation of many electronic devices. Shields are used to either expel fields from an area or to confine fields to a region in, e.g., superconducting quantum interface devices for precision measurements of magnetic fields [1], magnetic resonance imaging machines and tokamaks in magnetic confinement fusion $[2,3]$. Further more, electromagnetic shields are commonly used in advanced nanotechnology research facilities, biomedical research laboratories, continuous beam accelerators and various facilities such as transformer vault and switchgear in electrical-power industry $[4,5]$. For frequencies above $100 \mathrm{kHz}$, satisfactory shielding performance can be achieved by a conductive shell (Faraday shield), which makes use of the eddy current to absorb the electromagnetic waves [6]. For a low-frequency electromagnetic wave or static electric or magnetic field, shields made of high magnetic permeability metal alloys can be used, such as sheets of $\mu$-metal, to channel flux lines around the shielded region $[7,8,9,10]$. The shielding effectiveness depends on the permeability, which generally drops off at both very low magnetic field strengths and at high field strengths where the material becomes saturated. So to achieve low residual fields, magnetic shields often consist of several enclosures one inside the other, each of which successively reduces the inner field $[11,12$, 13, 14]. Also, superconducting materials can expel magnetic fields by the Meissner effect. However, a single shield with relative permeability of $0 \leq \mu_{r}<1$ (diamagnetic) or $\mu_{r}>1$ (paramagnetic, ferromagnetic) disturbs the external magnetic field in free space.

There are many situations in which it may be desirable to hide a sensitive object from a magnetic field, but not to disturb the applied field. In this way, external functions of the field, such as resolving objects in a magnetic resonance imaging scan, are preserved. A direct current magnetic cloak that shields the inner region without affecting the exterior space requires an inhomogeneous and anisotropic medium characterized by a response that is diamagnetic in one direction and paramagnetic in the perpendicular directions $[15,16]$, as demanded by transformation optics [17] in the limit of zero frequency. These specifications were met in an experiment for a cylindrical cloak [18] using a novel design for the required diamagnetic metamaterials [19]. Also magnetic cloaking of uniform static fields has been realized using a superconducting-ferromagnetic bilayer [20].

In this paper, we develop a method for designing magnetic shields made of isotropic materials that do not perturb applied multipole fields in the static regime. The idea is inspired by the concept of neutral inclusion in the theory of composites with respect to a uniform field [21, 22, 23, 24, 25]. Another motivation comes from the theoretical proposals and experimental efforts to realize cloaking by manipulating various materials $[17,26,27,28,29,30]$. Perfect cloaking requires that the shield and the object we wish to hide do not disturb the wave field in the exterior domain for an incident wave from any source. This means that the solution to Maxwell's equations remains exactly the same in the exterior domain as if we use the vacuum permittivity and permeability everywhere in space. Such a requirement is rather stringent but can be met by using materials with their physical moduli equal to negative numbers, zero or infinite, which might be realized by metamaterials. An approximate cylindrical cloak for microwaves has been realized from a reduced set of effective anisotropic and inhomogeneous material parameters [31]. Relaxing the requirement of perfect cloaking one can use plasmonic covers to cancel the dominant scattering from an object [32]. In this approach there is no need for anisotropy and several layers can provide a discrete inhomogeneity to obtain multifrequency solutions [33]. In the quasi-static limit the dipole case is nothing but the transparency condition for a uniform field [34].

We present a formula for making cylindrical coreshell structures with concentric layers, characterized by isotropic and homogeneous permeabilities, neutral to applied multipole fields (section 3). In the limit of a perfect diamagnetic (or an infinite $\mu$-metal) response of the innermost layer we find exact cloaking solutions for every multipole order. This is an extensions of the double-layer dipole cloak [20] and every layer added to this structure constitutes an extra degree of freedom to obtain simultaneous cloaking solutions. The theory is illustrated with numerical simulations for a few-layer shields (section 4) and their significance in the problem of cloaking is discussed as we conclude (section 5). Next we describe the methodology (section 2). 


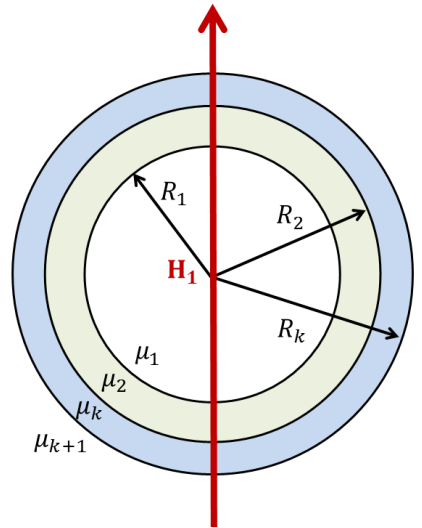

Figure 1. Schematic picture of a uniform magnetic field $\mathbf{H}_{1}$ over concentric cylindrical domains with isotropic relative permeabilities $\mu_{1}, \mu_{2}, \ldots, \mu_{k}$ in free space $\left(\mu_{k+1}=1\right)$ and radii $R_{1}, R_{2}, \ldots, R_{k}$ centered at the origin.

\section{Methodology}

We consider cylindrical core-shell structures with concentric layers made of materials with homogeneous and isotropic permeabilities. The exterior region is considered to be free space with the vacuum permeability $\mu_{0}$. The core can be either diamagnetic, paramagnetic (ferromagnetic) or be free space. If there are no currents present, the magnetic field $\mathbf{H}$ can be written in terms of a magnetic scalar potential $\psi$ as $\mathbf{H}=-\nabla \psi$. Using the consecutive relation for the magnetic flux density $\mathbf{B}=\mu \mathbf{H}$, the potential satisfies

$\nabla \cdot(\mu \nabla \psi)=0$

where $\mu$ is the permeability. In polar coordinates $(r, \theta)$ the potential can be expanded as

$\psi^{(j)}=\sum_{n=1}^{\infty} \psi_{n}^{(j)}(r, \theta)=\sum_{n=1}^{\infty}\left(A_{n}^{(j)} r^{n}+\frac{B_{n}^{(j)}}{r^{n}}\right) \cos n \theta$

in each medium with relative permeability $\mu_{j}=\mu / \mu_{0}$ for $j=1,2, \ldots, k, k+1$, see figure 1 . The number of layers in the shell is then $k-1$. The coefficients $A_{n}^{(j)}$ and $B_{n}^{(j)}$ are determined by the boundary conditions. By enforcing continuity of the potential (2) and of the normal component of the magnetic flux density $\mathbf{B}=-\mu \nabla \psi$ at the interface $r=R_{j}$ between $\mu_{j}$ and $\mu_{j+1}$, the boundary conditions can be expressed as

$$
\begin{aligned}
& {\left[\begin{array}{cc}
R_{j}^{2 n} & 1 \\
\mu_{j} & -\mu_{j} R_{j}^{-2 n}
\end{array}\right]\left[\begin{array}{l}
A_{n}^{(j)} \\
B_{n}^{(j)}
\end{array}\right]} \\
& =\left[\begin{array}{cc}
R_{j}^{2 n} & 1 \\
\mu_{j+1} & -\mu_{j+1} R_{j}^{-2 n}
\end{array}\right]\left[\begin{array}{l}
A_{n}^{(j+1)} \\
B_{n}^{(j+1)}
\end{array}\right] .
\end{aligned}
$$

For non-zero $\mu_{j}$ the matrix equation can be rewritten as

$$
\left[\begin{array}{l}
A_{n}^{(j)} \\
B_{n}^{(j)}
\end{array}\right]=T_{j}\left[\begin{array}{l}
A_{n}^{(j+1)} \\
B_{n}^{(j+1)}
\end{array}\right], \quad j=1,2, \ldots, k .
$$

The coefficients are related by the transfer matrix

$T_{j}=\frac{1}{1+\Gamma_{j}}\left[\begin{array}{cc}1 & \Gamma_{j} R_{j}^{-2 n} \\ \Gamma_{j} R_{j}^{2 n} & 1\end{array}\right]$,

where we have defined the mismatch factor

$\Gamma_{j}=\frac{\mu_{j}-\mu_{j+1}}{\mu_{j}+\mu_{j+1}}$.

This factor takes values in the range $-1 \leq \Gamma_{j} \leq 1$. The relation between the coefficients interior and exterior to a $(k-1)$-layer shield is found by iteration of (4) as

$\left[\begin{array}{c}A_{n}^{(1)} \\ B_{n}^{(1)}\end{array}\right]=\prod_{j=1}^{k} T_{j}\left[\begin{array}{c}A_{n}^{(k+1)} \\ B_{n}^{(k+1)}\end{array}\right]$.

We consider $A_{n}^{(k+1)}$ as given in terms of an applied field strength $H_{n}=\left|\nabla \psi_{n}^{(k+1)}\right|=n r^{n-1}\left|A_{n}^{(k+1)}\right|$ in the limit $r \rightarrow \infty$, originating from a magnetic multipole of order $n$ at infinity. The monopole $(n=0)$ term is zero implying that $A_{0}^{(k+1)}$ is an unphysical constant taken to be zero, which is why we consider $n \geq 1$ in the potential $(2)$. The dipole $(n=1)$ case corresponds to the uniform field in figure 1 . Due to the fact that the potential must be finite within the core $r<R_{1}$, we have $B_{n}^{(1)}=0$. Neutral shielding of each multipole field $\mathbf{H}_{n}=-\nabla \psi_{n}^{(k+1)}$ separately is found by equating (7) to $B_{n}^{(k+1)}=0$. This means that $T_{1} T_{2} \cdots T_{k}$ is an upper triangular matrix. We treat a few-layer shields in some detail and give a formula relating the permeabilities in the general case.

\section{Analytical results}

\subsection{Single-layer shields}

For one-layer shields the neutrality condition applied to equation (7) with $k=2\left(R_{k}=R_{2}\right)$ using (5) is

$\Gamma_{2}=-\Gamma_{1}\left(\frac{R_{1}}{R_{2}}\right)^{2 n}$,

where the allowed values of the mismatch factors $\Gamma_{1}$ and $\Gamma_{2}$ are determined by requiring that the ratio $R_{1} / R_{2}$ is less than unity. By expanding $\Gamma_{1}$ and $\Gamma_{2}$ using (6), we arrive at the permeability relation

$$
\mu_{3}=\mu_{2}+\frac{2 \mu_{2}\left(\mu_{1}-\mu_{2}\right)\left(\frac{R_{1}}{R_{2}}\right)^{2 n}}{2 \mu_{2}+\left(\mu_{1}-\mu_{2}\right)\left[1-\left(\frac{R_{1}}{R_{2}}\right)^{2 n}\right]} .
$$

This formula is exactly the same as the transparency condition for Mie scattering on an electrically small coated disc in the quasi-static limit [32]. We note that the exterior permeability $\mu_{3}$ can be seen as the effective permeability of the coated inclusion with respect to an arbitrary multipolar field of order $n \geq 1$. For a uniform field $(n=1)$ the effective permeability is equivalent to the Maxwell Garnet mixing rule for the composite with the area fraction $\left(R_{1} / R_{2}\right)^{2}$. 


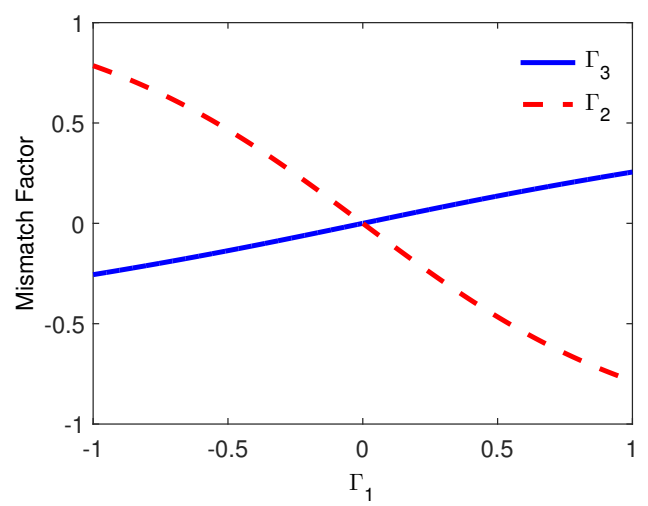

Figure 2. The mismatch factors $\Gamma_{3}$ and $\Gamma_{2}$ as functions of $\Gamma_{1}$ giving neutral shielding (10) of dipole $(n=1)$ and quadrupole $(n=2)$ fields for $R_{1} / R_{2}=0.75$ and $R_{2} / R_{3}=0.8$.

\subsection{Double-layer shields}

For two-layer shields the neutrality condition applied to equation (7) with $k=3$ using (5) reads

$\Gamma_{3}=-\frac{\Gamma_{2}+\Gamma_{1}\left(\frac{R_{1}}{R_{2}}\right)^{2 n}}{1+\Gamma_{2} \Gamma_{1}\left(\frac{R_{1}}{R_{2}}\right)^{2 n}}\left(\frac{R_{2}}{R_{3}}\right)^{2 n}$.

The allowed values of the mismatch factors $\Gamma_{1}, \Gamma_{2}$ and $\Gamma_{3}$ are determined by requiring that the ratios $R_{1} / R_{2}$ and $R_{2} / R_{3}$ are positive and less than unity. For a given geometry the mismatch factors $\Gamma_{2}$ and $\Gamma_{3}$ can be uniquely determined as functions of $\Gamma_{1}$; equating the mismatch factor $\Gamma_{3}(n=1)$ to $\Gamma_{3}(n=2)$ in (10) yields a quadratic equation in $\Gamma_{2}$, which admits one acceptable $\left(\left|\Gamma_{2}\right| \leq 1\right)$ analytical solution. This solution and the corresponding mismatch factor $\Gamma_{3}$ are plotted in figure 2 as functions of $\Gamma_{1}$ for the thickness ratios $R_{1} / R_{2}=0.75$ and $R_{2} / R_{3}=0.8$. We observe that $\Gamma_{2}$ is a decreasing function of $\Gamma_{1}$, whilst $\Gamma_{3}$ is an increasing function of $\Gamma_{1}$, and that they have opposite signs. A magnetic shield satisfying these conditions is neutral to both dipole $(n=1)$ and quadrupole $(n=2)$ fields. The shielding effectiveness is commonly quantified by the shielding factor, $S$, which is defined by the ratio of the magnetic field without the shield to the magnetic field with the shield at the same point. The shielding factor is unity for complete transparency and turns to infinity in the limit of perfect screening. Here, we consider the inverse of the shielding factor in order to get values in the unit interval. Solving equation (7) for $B_{n}^{(1)}=B_{n}^{(4)}=0$ using (10) we find the ratio

$\frac{A_{n}^{(1)}}{A_{n}^{(4)}}=\frac{\left(1-\Gamma_{1}\right)\left(1-\Gamma_{2}\right)}{\left(1+\Gamma_{3}\right)\left[1+\Gamma_{2} \Gamma_{1}\left(\frac{R_{1}}{R_{2}}\right)^{2 n}\right]}$

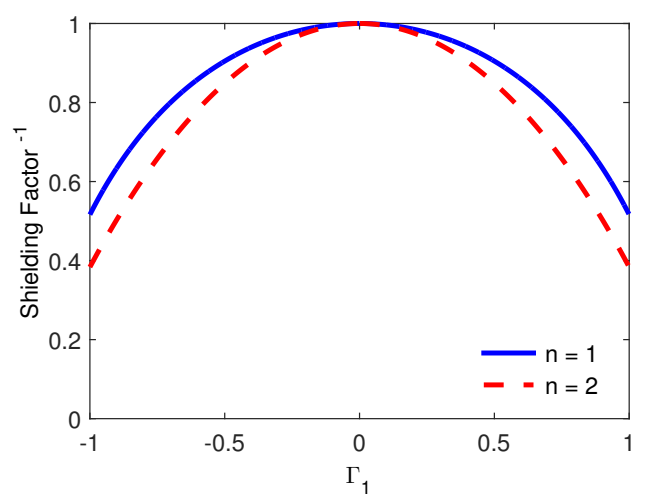

Figure 3. The inverse shielding factor (13) for $n=1,2$ as functions of $\Gamma_{1}$ using $R_{1} / R_{2}=0.75$. The mismatch factors $\Gamma_{2}$ and $\Gamma_{3}$ are those in figure 2 giving neutrality to both dipole and quadrupole fields.

of the core coefficient $A_{n}^{(1)}$ to the exterior coefficient $A_{n}^{(4)}$. Removing the shield we get the ratio

$\frac{\widetilde{A}_{n}^{(1)}}{A_{n}^{(4)}}=\frac{2 \mu_{4}}{\mu_{1}+\mu_{4}}$

of the core coefficient $\widetilde{A}_{n}^{(1)}$ to the exterior coefficient $A_{n}^{(4)}$. (Formally this follows from the coefficient relation (11) by putting $\mu_{2}=\mu_{3}=\mu_{4}$ so that $1-\Gamma_{1}=$ $2 \mu_{4} /\left(\mu_{1}+\mu_{4}\right)$ and $\Gamma_{2}=\Gamma_{3}=0$.) Without a shield the core scatters the applied field unless $\mu_{1}=\mu_{4}$ in which case (12) is unity. The inverse of the shielding factor can be expressed as

$S^{-1}=\frac{A_{n}^{(1)}}{\widetilde{A}_{n}^{(1)}}=\frac{1+\Gamma_{2} \Gamma_{1}+\Gamma_{3}\left(\Gamma_{1}+\Gamma_{2}\right)}{\left(1-\Gamma_{3}^{2}\right)\left[1+\Gamma_{2} \Gamma_{1}\left(\frac{R_{1}}{R_{2}}\right)^{2 n}\right]}$,

taking the value 1 for complete transparency and 0 for perfect screening. This expression follows from equations (11) and (12) with repeated use of the mismatch factors (6). In figure 3 we plot $S^{-1}$ for $n=1,2$ as functions of $\Gamma_{1}$ using $R_{1} / R_{2}=0.75$ and the mismatch factors in figure 2 . The inverse shielding factor (13) clearly decreases with increasing order $n$ since $\Gamma_{1}$ and $\Gamma_{2}$ have opposite signs and $\left(R_{1} / R_{2}\right)^{n}$ decreases with increasing value of $n$. The larger the absolute value of $\Gamma_{1}$ the better the shielding.

By expanding $\Gamma_{2}$ and $\Gamma_{3}$ in equation (10) using (6), we find the permeability relation for the two-layer shield to be

$\mu_{4}=\mu_{3}+\frac{2 \mu_{3}\left(\mu_{2}^{\star}-\mu_{3}\right)\left(\frac{R_{2}}{R_{3}}\right)^{2 n}}{2 \mu_{3}+\left(\mu_{2}^{\star}-\mu_{3}\right)\left[1-\left(\frac{R_{2}}{R_{3}}\right)^{2 n}\right]}$, 

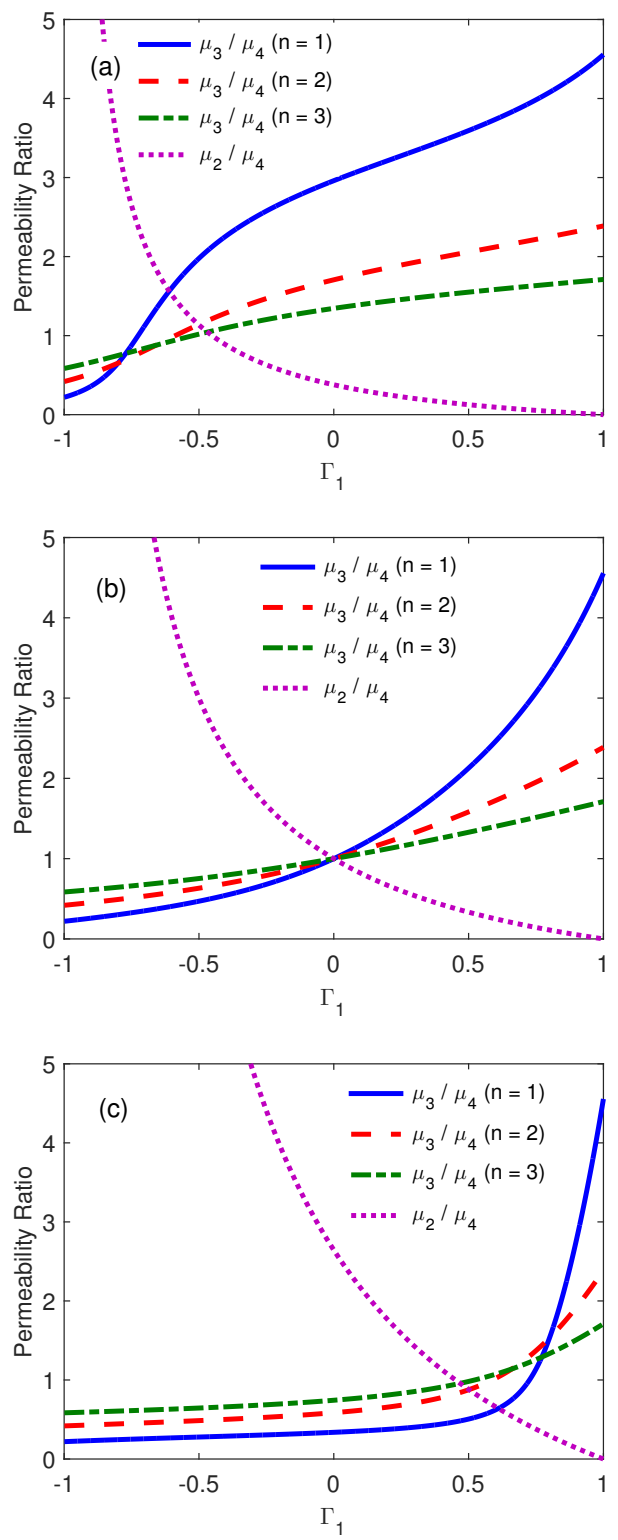

Figure 4. The permeability ratios $\mu_{2} / \mu_{4}$ and $\mu_{3} / \mu_{4}$ for neutral shielding of dipole $(n=1)$, quadrupole $(n=2)$, and octupole $(n=3)$ fields as functions of $\Gamma_{1}$ in the case (a) $\mu_{1} / \mu_{4}=0.3782$, (b) $\mu_{1} / \mu_{4}=1$ and (c) $\mu_{1} / \mu_{4}=2.6444$. The thickness ratios are $R_{1} / R_{2}=0.75$ and $R_{2} / R_{3}=0.8$.

where

$$
\mu_{2}^{\star}=\mu_{2}+\frac{2 \mu_{2} \Gamma_{1}\left(\frac{R_{1}}{R_{2}}\right)^{2 n}}{1-\Gamma_{1}+\Gamma_{1}\left[1-\left(\frac{R_{1}}{R_{2}}\right)^{2 n}\right]}
$$

denotes the effective permeability (9) of the single-layer shield, expressed in terms of the mismatch factor $\Gamma_{1}$, the ratio $R_{1} / R_{2}$, and the relative permeability

$\mu_{2}=\mu_{1} \frac{1-\Gamma_{1}}{1+\Gamma_{1}}$.

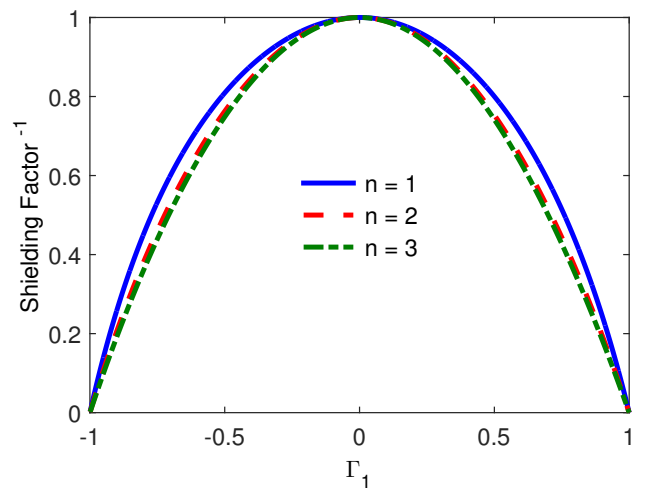

Figure 5. The inverse shielding factor (13) with $\mu_{1} / \mu_{4}=1$ and $R_{1} / R_{2}=0.75$ for $n=1,2,3$ as functions of $\Gamma_{1}$. The permeabilities $\mu_{2}$ and $\mu_{3}$ are those in figure $4(\mathrm{~b})$ giving neutral shielding of each multipole field separately.

The permeability relation (14) means that a multipole field in the exterior region $\left(r>R_{3}\right)$ is not perturbed by the core-shell structure $\left(0<r<R_{3}\right)$, which forms an inclusion in the background with $\mu_{4}$ as the effective permeability. Figure 4 shows the permeability ratios $\mu_{3} / \mu_{4}$ in (14) and $\mu_{2} / \mu_{4}$ in (16) for neutral shielding of dipole $(n=1)$, quadrupole $(n=2)$ and octupole $(n=3)$ fields as functions of $\Gamma_{1}$ for three characteristic values of $\mu_{1} / \mu_{4}$ using the thickness ratios $R_{1} / R_{2}=0.75$ and $R_{2} / R_{3}=0.8$. When $\mu_{1} / \mu_{4}<1$ (as in figure $4(\mathrm{a})$ ) the curves (14) for two different $n$ intersect in the range $-1<\Gamma_{1}<0$, corresponding to simultaneous solutions at a specific permeability ratio $\mu_{3} / \mu_{4}<1$. When $\mu_{1} / \mu_{4}>1$ (as in figure $4(\mathrm{c})$ ) the curves (14) for two different $n$ intersect in the range $0<\Gamma_{1}<1$, corresponding to simultaneous solutions at a specific permeability ratio $\mu_{3} / \mu_{4}>1$. When $\mu_{1} / \mu_{4}=1$ (figure $4(\mathrm{~b})$ ) all curves intersect each other at $\Gamma_{1}=0$, corresponding to the trivial solution $\mu_{1}=\mu_{2}=\mu_{3}=\mu_{4}$. However, separate multipole solutions for neutral shielding of the core exist for nontrivial material parameters. In this case there is no scattering when the shield is removed and consequently the inverse of the corresponding shielding factors become zero in the limits $\Gamma_{1} \rightarrow 1\left(\Gamma_{2} \rightarrow-1\right)$ and $\Gamma_{1} \rightarrow-1\left(\Gamma_{2} \rightarrow 1\right)$ as shown in figure 5 . In these limits we can place any object in the central region without affecting the shielding effectiveness.

In the limit $\mu_{2} \rightarrow 0\left(\mu_{2}^{\star} \rightarrow 0\right)$, the permeability relation (14) reduces to

$\frac{\mu_{3}}{\mu_{4}}=\frac{1+\left(\frac{R_{2}}{R_{3}}\right)^{2 n}}{1-\left(\frac{R_{2}}{R_{3}}\right)^{2 n}}$.

This gives perfect screening of the core and thus defines a cloaking solution for the applied multipole field of order $n \geq 1$, depending on the ratios $\mu_{3} / \mu_{4}$ and $R_{2} / R_{3}$. 


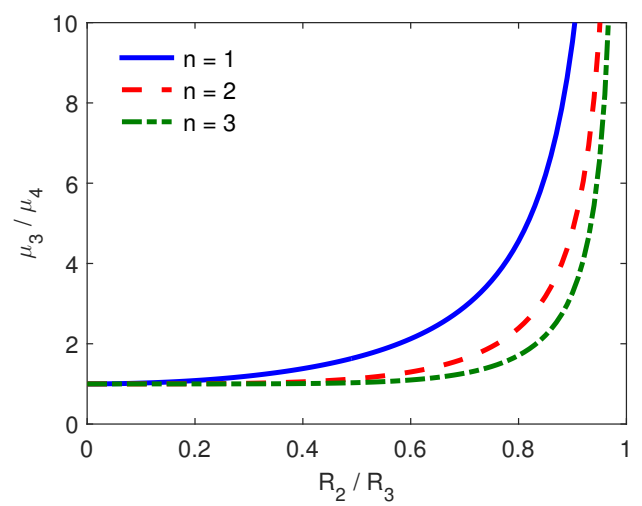

Figure 6. The permeability ratio $\mu_{3} / \mu_{4}$ as a function of $R_{2} / R_{3}$ in the case $\mu_{2}=0$ for cloaking of dipole $(n=1)$, quadrupole $(n=2)$ and octupole $(n=3)$ fields, respectively.

Figure 6 shows the ratio (17) as a function of $R_{2} / R_{3}$ for cloaking of dipole $(n=1)$, quadrupole $(n=2)$ and octupole $(n=3)$ fields, respectively. This formula is an extension of the dipole case [20] to multipolar fields. For $R_{2} / R_{3}=0.8$ the values $(17)$ can also be read at $\Gamma_{1}=1$ in figure 4 and both $\mu_{3} / \mu_{4}=4.5556$ $(n=1)$ and $\mu_{3} / \mu_{4}=2.3875(n=2)$ will be used in the numerical simulations. A perfect diamagnetic response of the inner layer $\left(\mu_{2}=0\right)$ corresponds to $\Gamma_{1}=-\Gamma_{2}=1$ in which case equation (10) reduces to $\Gamma_{3}=\left(R_{2} / R_{3}\right)^{2 n}$, being identical to the expression (17). In the limit $\mu_{2} \rightarrow \infty\left(\mu_{2}^{\star} \rightarrow \infty\right)$, we obtain

$\frac{\mu_{3}}{\mu_{4}}=\frac{1-\left(\frac{R_{2}}{R_{3}}\right)^{2 n}}{1+\left(\frac{R_{2}}{R_{3}}\right)^{2 n}}$,

which is another cloaking solution of order $n \geq 1$. The permeability ratio (18) is inverted as compared to (17) and the values are read at $\Gamma_{1}=-1$ in figure 4 for the thickness ratio $R_{2} / R_{3}=0.8$. This solution can also be found directly from the neutrality condition (10). The limit $\mu_{2} \rightarrow \infty$ corresponds to $\Gamma_{1}=-\Gamma_{2}=-1$ in which case equation (10) reduces to $\Gamma_{3}=-\left(R_{2} / R_{3}\right)^{2 n}$, being identical to the expression (18). Hence doublelayer shields support unique cloaking solutions for every multipole field, that is, there are no concurrent solutions since the curves never intersect each other.

\subsection{Multi-layer shields}

In general, we find that neutral $(k-1)$-layer shields can be calculated iteratively by putting the exterior permeability $\mu_{k+1}$ equal to the effective permeability (with respect to a multipole field of order $n \geq 1$ )

$$
\mu_{k}^{\star}=\mu_{k}+\frac{2 \mu_{k}\left(\mu_{k-1}^{\star}-\mu_{k}\right)\left(\frac{R_{k-1}}{R_{k}}\right)^{2 n}}{2 \mu_{k}+\left(\mu_{k-1}^{\star}-\mu_{k}\right)\left[1-\left(\frac{R_{k-1}}{R_{k}}\right)^{2 n}\right]}
$$

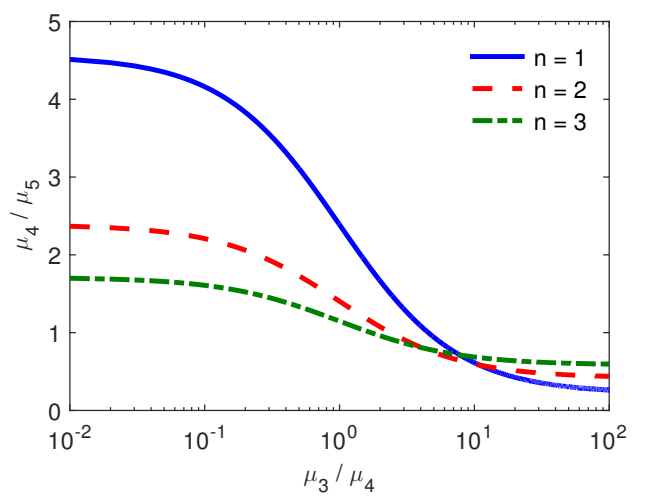

Figure 7. The permeability ratio $\mu_{4} / \mu_{5}$ as a function of $\mu_{3} / \mu_{4}$ for cloaking of dipole $(n=1)$, quadrupole $(n=2)$ and octupole $(n=3)$ fields. The thickness ratios are $R_{2} / R_{3}=R_{3} / R_{4}=0.8$.

of the core-shell structure for $k \geq 2$. Here $\mu_{k}$ is the permeability of the $k$ th domain, $R_{k-1} / R_{k}$ is the corresponding thickness ratio and $\mu_{k-1}^{\star}$ is the effective permeability of the $(k-2)$-layer shield, taking $\mu_{1}^{\star}$ to be the core permeability $\mu_{1}$. This is an extension of a neutral cylindrical coated inclusion [23] from uniform to multipolar fields. As an example, we consider the three-layer shield defined by confining $\mu_{4}$ to the layer $R_{3}<r<R_{4}$ in figure 1 and introducing $\mu_{5}$ as the exterior permeability. In the case of zero permeability of the innermost layer, $\mu_{2}=0$, we obtain

$$
\frac{\mu_{5}}{\mu_{4}}=1+\frac{2\left[\frac{\mu_{3}}{\mu_{4}} \frac{1-\left(\frac{R_{2}}{R_{3}}\right)^{2 n}}{1+\left(\frac{R_{2}}{R_{3}}\right)^{2 n}}-1\right]\left(\frac{R_{3}}{R_{4}}\right)^{2 n}}{2+\left[\frac{\mu_{3}}{\mu_{4}} \frac{1-\left(\frac{R_{2}}{R_{3}}\right)^{2 n}}{1+\left(\frac{R_{2}}{R_{3}}\right)^{2 n}}-1\right]\left[1-\left(\frac{R_{3}}{R_{4}}\right)^{2 n}\right]},
$$

using $\mu_{4}^{\star}=\mu_{5}$ in (19) and $\mu_{3}^{\star}=\mu_{4}$ in (17) for the effective permeabilities. The permeability ratio $\mu_{4} / \mu_{5}$ is plotted versus $\mu_{3} / \mu_{4}$ in figure 7 for cloaking of dipole $(n=1)$, quadrupole $(n=2)$ and octupole $(n=3)$ fields, respectively, using the geometry $R_{2} / R_{3}=0.8$, $R_{3} / R_{4}=0.8$. Curves corresponding to different orders intersect each other once. The intersection points can be found analytically by solving (20) for the order pairs. For example, the dipole and quadrupole curves in figure 7 intersect each other at $\left(\mu_{3} / \mu_{4}, \mu_{4} / \mu_{5}\right)=$ $(10,8765,0.5846)$. Taking $\mu_{5}=1$ the layers alternate between being diamagnetic and paramagnetic for the simultaneous solutions. The values of the permeability ratio $\mu_{4} / \mu_{5}$ in the limits $\mu_{3} \rightarrow 0$ and $\mu_{3} \rightarrow \infty$ happen to coincide with those of (17) and (18), respectively, due to the equal thickness ratios $R_{3} / R_{4}=R_{2} / R_{3}=$ 0.8 . The cloaking solution (20) can also be expressed 
in terms of mismatch factors as

$$
\Gamma_{4}=\frac{\Gamma_{3}-\left(\frac{R_{2}}{R_{3}}\right)^{2 n}}{\Gamma_{3}\left(\frac{R_{2}}{R_{3}}\right)^{2 n}-1}\left(\frac{R_{3}}{R_{4}}\right)^{2 n},
$$

which is the neutrality condition obtained from (7) with $k=4$ using (5) in the case $\Gamma_{1}=-\Gamma_{2}=1$. For a given geometry the relation (21) defines cloaking solutions of order $n$ with two degrees of freedom, constituted by the mismatch factors $\Gamma_{3}$ and $\Gamma_{4}$. One extra degree of freedom is provided by every layer (mismatch factor) added to the magnetic shields which may be exploited to cloak additional multipole fields.

\section{Numerical simulations}

We illustrate the results by numerical simulations.

\subsection{Neutral shielding}

In figure 8 we illustrate the neutral performance of two-layer shields with thickness ratios $R_{1} / R_{2}=0.75$ and $R_{2} / R_{3}=0.8$ using COMSOL Multiphysics. A uniform magnetic field is applied in the surrounding medium with relative permeability $\mu_{4}=1$. The horizontal lines are field lines and the color tables show the absolute values of the magnetic flux densities for an implementation of a diamagnetic core with $\Gamma_{1}=-0.8\left(\mu_{1}=0.3782, \mu_{2}=3.4034, \mu_{3}=0.6526\right)$ in figure $8(\mathrm{a})$ and a paramagnetic core with $\Gamma_{1}=$ $0.8\left(\mu_{1}=2.6444, \mu_{2}=0.2938, \mu_{3}=1.5323\right)$ in figure $8(\mathrm{c})$. The material parameters used come from the analytical expressions (14) and (16). The neutral shielding performance is excellent in the dipole $(n=1)$ case and it should apply also to a quadrupole $(n=2)$ field since the numerical simulations correspond to the intersections of the dipole and quadrupole curves at $\Gamma_{1}=-0.8$ and $\Gamma_{1}=0.8$ in figure $4(\mathrm{a})$ and figure $4(\mathrm{c})$, respectively. For simultaneous solutions either the core is diamagnetic $\left(0<\mu_{1}<1\right)$, the inner layer paramagnetic $\left(\mu_{2}>1\right)$ and the outer layer diamagnetic $\left(0<\mu_{3}<1\right)$ or the types of responses are interchanged so that the core is paramagnetic, the inner layer diamagnetic and the outer layer paramagnetic. The only common solution for a vacuum core $\left(\mu_{1}=1\right)$ is the trivial one with $\mu_{2}=\mu_{3}=1$ at $\Gamma_{1}=0$ in figure $4(\mathrm{~b})$. However, separate multipole solutions for neutral shielding of the core exist for nontrivial material parameters. Simulation results of a vacuum core implementation with $\Gamma_{1}=0.8\left(\mu_{1}=1, \mu_{2}=\right.$ $\left.0.1111, \mu_{3}=3.3000\right)$ is shown in figure $8(\mathrm{~b})$ in the dipole case. One may note that the theory relies on the core to have homogeneous permeability. If we were to place an object in the vacuum core, such as a circuit board that needs to be shielded from external magnetic fields, the neutral shielding would (a)

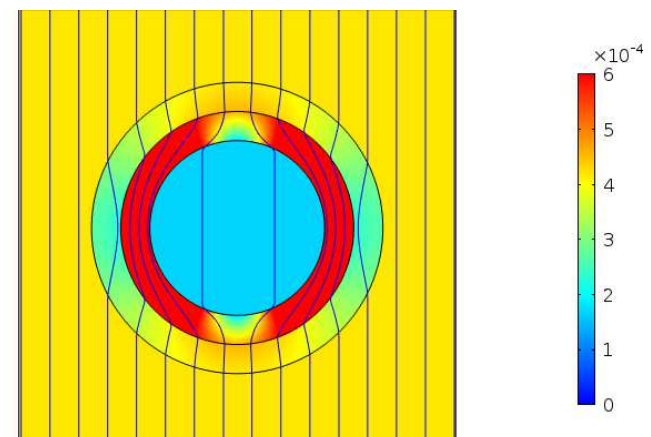

(b)

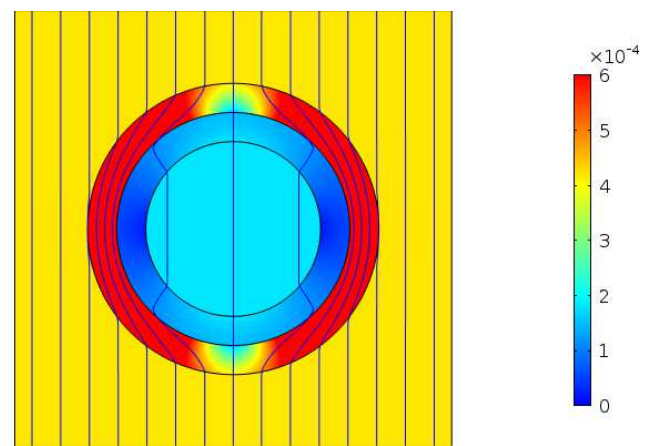

(c)

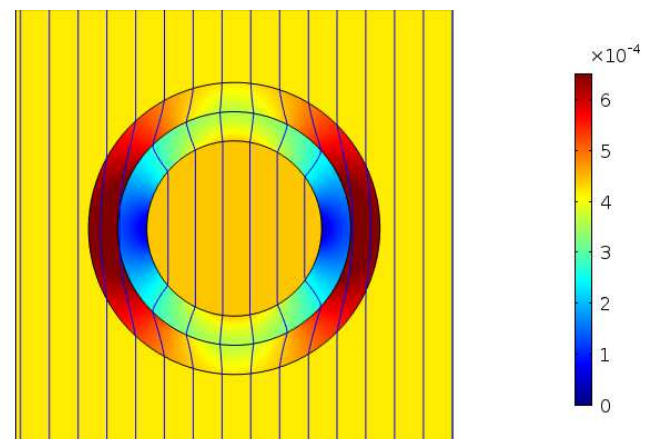

Figure 8. Simulation results of neutral magnetic shields with thickness ratios $R_{1} / R_{2}=0.75$ and $R_{2} / R_{3}=0.8$ using COMSOL Multiphysics. The surrounding medium is vacuum with relative permeability $\mu_{4}=1$ where a uniform magnetic field is applied. Field lines are drawn and the color tables show the absolute values of the magnetic flux densities for (a) a diamagnetic core with $\Gamma_{1}=-0.8\left(\mu_{1}=0.3782, \mu_{2}=3.4034, \mu_{3}=0.6526\right)$, (b) a vacuum core with $\Gamma_{1}=0.8\left(\mu_{1}=1, \mu_{2}=0.1111, \mu_{3}=3.3000\right)$ and (c) a paramagnetic core with $\Gamma_{1}=0.8\left(\mu_{1}=2.6444\right.$, $\left.\mu_{2}=0.2938, \mu_{3}=1.5323\right)$.

be destroyed unless the layer permeabilities satisfy the cloaking solution (17) or (18). In order to illustrate neutral shielding and cloaking of multipole fields we invoke a transformation.

\subsection{A conformal mapping}

A uniform magnetic field with strength $H_{1}$ at the origin, which is transverse to the cylindrical core-shell structure and is invariant over the cross section in figure 1 can be though of as originating from a magnetic 


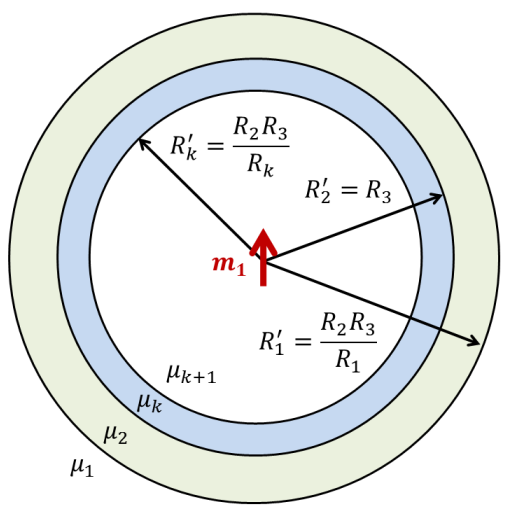

Figure 9. Applying the conformal mapping (22) to the problem in figure 1 gives the complementary problem with a magnetic dipole $\mathbf{m}_{1}$ at the origin and relative permeabilities $\mu_{j}^{\prime}=\mu_{k+2-j}$ for $j=1,2, \ldots, k, k+1$.

dipole at infinity. Applying the transformation

$r^{\prime}=R_{2} R_{3} / r, \quad \theta^{\prime}=\theta$,

turns the geometry inside out in that it maps the circle $R_{2}$ to the circle $R_{3}$, the origin to infinity and vice versa. Hence the magnetic dipole at infinity, which created the uniform field, is transformed to a magnetic dipole at the origin, see figure 9. Any potential (2) that is a valid solution to the boundary value problem (1) is also a solution to the transformed configuration due to invariance of the Laplace equation under the transformation (22). This result can be viewed as a basic application of transformation optics [17] at zero frequency ensuring that all material properties are maintained as isotropic. In view of the conformal mapping (22) the layer ordering $j$ is reversed such that $\mu_{j}^{\prime}=\mu_{k+2-j}$ and the individual radii $R_{j}^{\prime}$ are different, but the permeability values (19) remain invariant since the thickness ratios $R_{j}^{\prime} / R_{j-1}^{\prime}=R_{j-1} / R_{j}$ are preserved. Hence neutral field expulsion of nonuniform fields (applied at infinity) by magnetic shields and their performance can be visualized by neutral field confinement using magnetic multipoles at the origin.

\subsection{Cloaking}

Taking the limit $\mu_{2} \rightarrow 0$ of the two-layer neutral shield in figure $8(\mathrm{~b})$ with $R_{1} / R_{2}=0.75, R_{2} / R_{3}=0.8$ and $\mu_{1}=\mu_{4}=1$ yields the cloaking solution (17). Application of the conformal mapping (22) results in a cloak with the layer ordering reversed, i.e., $\mu_{2}^{\prime}=\mu_{3}$ and $\mu_{3}^{\prime}=\mu_{2}$, and the thickness ratios $R_{3}^{\prime} / R_{2}^{\prime}=R_{2} / R_{3}$ and $R_{2}^{\prime} / R_{1}^{\prime}=R_{1} / R_{2}$ preserved, see figure 9 . This configuration is implemented in COMSOL Multiphysics for numerical illustration of cloaking of a magnetic dipole in figure 10(a) and a quadrupole (realized by two magnetic dipoles) in figure $10(d)$ centered at the origin. Field lines are drawn and the color tables show the absolute values of the magnetic flux densities. The cloak's inner layer is paramagnetic with relative permeability $\mu_{3}=4.5556$ $(n=1)$ in figure $10(\mathrm{a})$ and $\mu_{3}=2.3875(n=$ 2 ) in figure $10(d)$, and the outer layer is a perfect diamagnet, $\mu_{2}=0$. For comparison, we calculate the fields without the cloaks by substituting the layers by the vacuum permeability and plot the differences between the magnetic flux densities with and without the cloak for the dipole excitation in figure 10(b) and the quadrupole excitation in figure 10(e). The differences in the cores are not identically zero but the neutral shielding performance is very good indeed. The discrepancy is attributed to the physical realization of the magnetic dipoles. The good performance becomes even more clear if the wrong shield is used with respect to the applied field. The latter is illustrated in figure $10(\mathrm{c})$ and figure $10(\mathrm{f})$ where the shields have been interchanged, i.e., $\mu_{3}(n=1) \leftrightarrow \mu_{3}(n=2)$ resulting in significant variations in the cores. This result confirms the fact that dipole and quadrupole fields cannot be simultaneously cloaked.

We exploit the degrees of freedom of a three-layer shield to illustrate that the structure supports simultaneous cloaking solutions of dipole and quadrupole fields as predicted by (20). In figure 11(a) and figure 11(d) we show simulation results of uniform field expulsion by a magnetic cloak with thickness ratios $R_{1} / R_{2}=0.75$ and $R_{2} / R_{3}=R_{3} / R_{4}=0.8$ in vacuum using COMSOL Multiphysics. Field lines are drawn and the color tables show the absolute values of the magnetic flux densities with the cloak (figure 11(a)) and the norm of the differences between the magnetic flux densities with and without the cloak (figure $11(\mathrm{~d})$ ). The interior and exterior medium has the vacuum permeability, $\mu_{1}=\mu_{5}=1$, and the layer closest to the shielded region is a perfect diamagnet $\left(\mu_{2}=0\right)$. The middle layer is paramagnetic with relative permeability $\mu_{3}=6.3584$ and the layer closest to the applied field is diamagnetic with relative permeability $\mu_{4}=0.5846$ corresponding to the intersection of the dipole $(n=1)$ and quadrupole $(n=2)$ curves at $\left(\mu_{3} / \mu_{4}, \mu_{4} / \mu_{5}\right)=(10,8765,0.5846)$ in figure 7 . Applying the conformal mapping (22) to figure 11 (a) results in a cloak with the layer ordering reversed but with the thickness ratios $R_{2}^{\prime} / R_{1}^{\prime}=0.75$ and $R_{4}^{\prime} / R_{3}^{\prime}=R_{3}^{\prime} / R_{2}^{\prime}=0.8$ preserved. In figure $11(\mathrm{~b})$ and figure $11(\mathrm{c})$ we show simulation results of neutral field confinement by exciting a dipole and a quadrupole centered at the origin, respectively. In this case no field lines permeate the perfect diamagnetic material of the outer screen. The results for the norm of the differences between the magnetic flux densities with and without the cloaks in figure 11(e) and figure 11(f) show good agreement with theory in that the interior fields are not perturbed by the cloaks. 

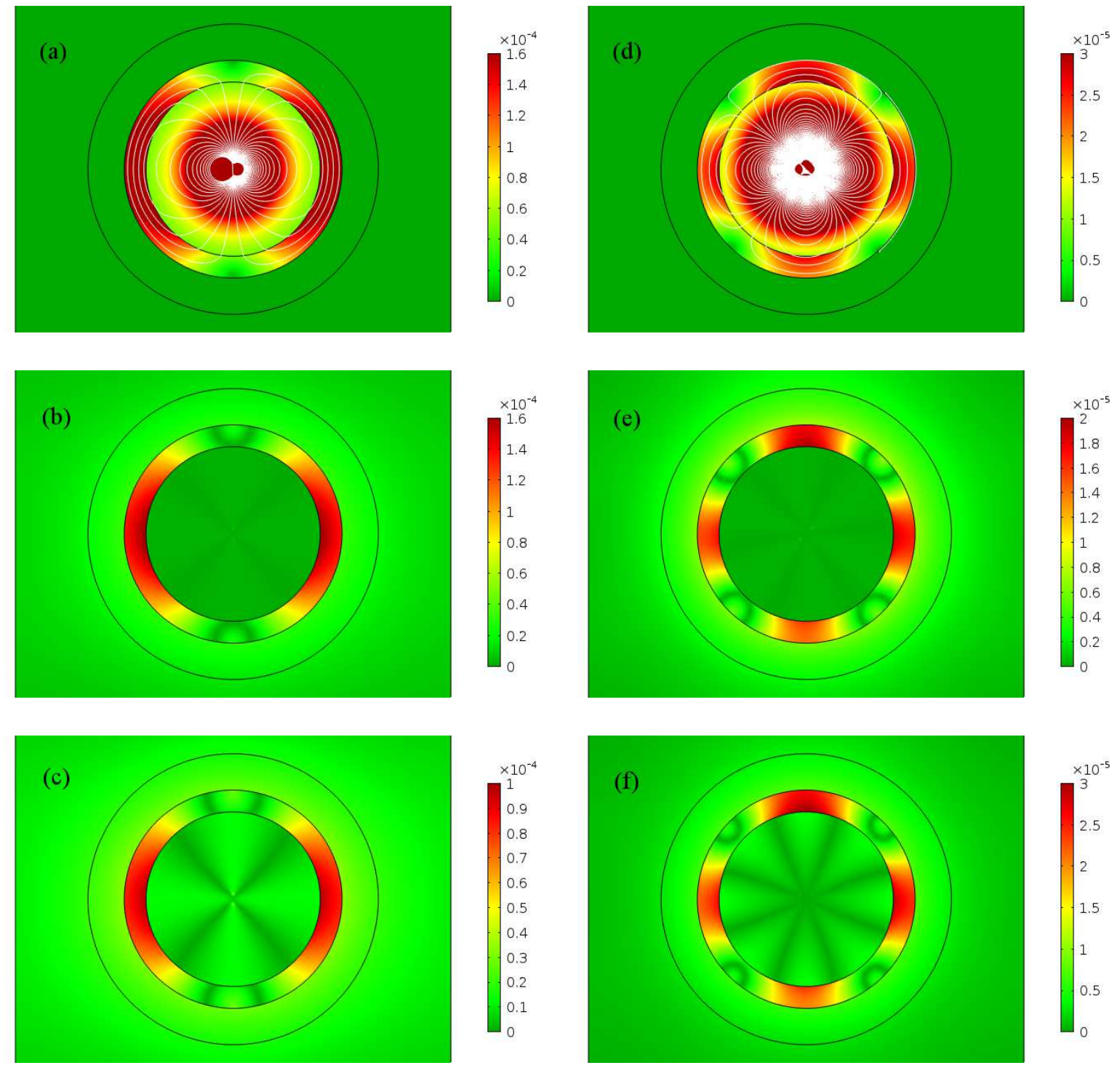

Figure 10. Simulation results of two-layer magnetic shields with thickness ratios $R_{3}^{\prime} / R_{2}^{\prime}=R_{2} / R_{3}=0.8$ and $R_{2}^{\prime} / R_{1}^{\prime}=R_{1} / R_{2}=0.75$ using COMSOL Multiphysics. The interior medium is vacuum with relative permeability $\mu_{4}=1$ where a magnetic dipole ((a)-(c)) or a quadrupole $((\mathrm{d})-(\mathrm{f}))$ is centered at the origin. Field lines are drawn in white and the color tables show the absolute values of the magnetic flux densities. The cloak's inner layer is paramagnetic with relative permeability $\mu_{3}(n=1)=4.5556((\mathrm{a})-(\mathrm{b}))$ or $\mu_{3}(n=2)=2.3875((\mathrm{~d})-(\mathrm{e}))$ and the outer layer is a perfect diamagnet, $\mu_{2}=0$. The exterior medium has the vacuum permeability, $\mu_{1}=1$. The norm of the differences between the magnetic flux densities with and without the shield ((b) and (e)) and the same but for interchanged shields ((c) and (f)).

\section{Discussion and conclusion}

In conclusion, we designed magnetic shields that do not perturb applied multipole fields in the static regime and evaluated their shielding effectiveness. Specifically, we presented an effective permeability relation for making cylindrical core-shell structures with concentric layers, characterized by isotropic and homogeneous permeabilities, neutral to arbitrary applied multipole fields from which cloaking solutions for every multipole order can be found. The two-layer cloaking formulas are generalizations of the dipole case [20]. By adding a third layer to the core-shell structure two concurrent multipole fields can be exactly cloaked. By extension, magnetic shields with $k$ layers support simultaneous cloaking solutions of $k-1$ different multipole fields. The recursive formula can be used to design the cloaks, which is a stationary case of the general cloaking problem for the particular geometry. The solutions are exact and thereby different from approximation methods such as homogenization [35, 36] or numerical optimization [37] of multilayered structures. However, to include every multipole field in the solution we have to let $k$ turn to infinity, resulting in an infinite series of 

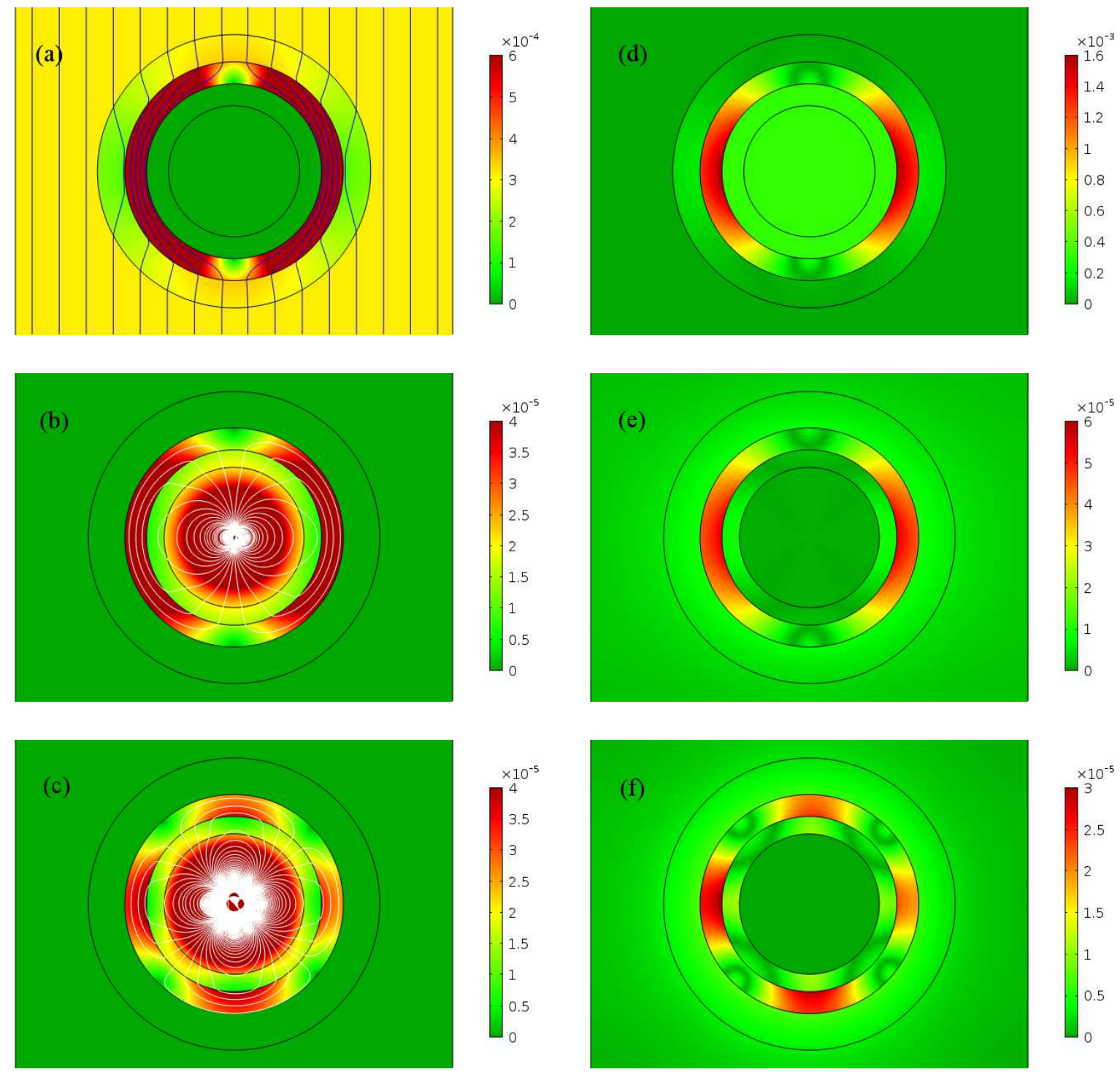

Figure 11. Simulation results of a three-layer magnetic cloak with thickness ratios $R_{1} / R_{2}=0.75$ and $R_{2} / R_{3}=R_{3} / R_{4}=0.8$ for uniform field expulsion ( (a) and (d)) and the complementary dipole field confinement cloak with thickness ratios $R_{2}^{\prime} / R_{1}^{\prime}=$ $R_{1} / R_{2}=0.75$ and $R_{4}^{\prime} / R_{3}^{\prime}=R_{3} / R_{4}=0.8=R_{2} / R_{3}=R_{3}^{\prime} / R_{2}^{\prime}((\mathrm{b})$ and (e)), being a cloak also for quadrupole fields ((c) and (f)), using COMSOL Multiphysics. The layers closest to the shielded regions are perfect diamagnets $\left(\mu_{2}=0\right)$, the middle layers are paramagnetic with relative permeability $\mu_{3}=6.3584$, and the layers closest to the applied fields are diamagnetic with relative permeability $\mu_{4}=0.5846$. The interior and exterior media have the vacuum permeability, $\mu_{1}=\mu_{5}=1$. Field lines are drawn and the color tables show the absolute values of the magnetic flux densities with the cloaks ( (a)-(c)) and the norm of the differences between the magnetic flux densities with and without the cloaks $((d)-(f))$.

alternating diamagnetic and paramagnetic layers. We expect that such a discrete inhomogeneity mimics the continuously varying anisotropic material parameters in the transformation optics recipe for a $2 \mathrm{D}$ cloak [31].

The presented analytical results can be adopted to phenomena described by the Laplace's equation. This form occurs in many situations of physics and cloaking, including static thermal cloaks [38, 39], direct current electric cloaks [40] and diffusive light cloaks [41]. We note that it is possible to derive analogous theoretical results for other canonical geometries, e.g., in spherical symmetry, following a similar methodology.

\section{References}

[1] Polushkin V, Buev A and Koch H 1994 Appl. Supercond. 2 $597-611$

[2] Tavernier S, Bogaert F V D and Lancker L V 1979 Nucl. Instrum. Methods $167391-398$

[3] Crozier S, Forbes L K and Doddrell D M 1998 Meas. Sci. Technol. 9113

[4] Gustavson T L, Bouyer P and Kasevich M A 1997 Phys. Rev. Lett. $782046-2049$

[5] Ohta H and Matsui T 2000 Phys. C 341 - 3482713 - 2714 
[6] Okazaki Y and Ueno K 1992 J. Magn. Magn. Mater. 112 $192-194$

[7] Mager A 1968 J. Appl. Phys. 39 1914-1914

[8] Mager A 1970 IEEE Trans. Magn. 6 67-75

[9] Baum E and Bork J 1991 J. Magn. Magn. Mater. 10169 $-74$

[10] Calvo E, Cerrada M, Gil-Botella I, Palomares C, Rodrguez I, Toral F and Verdugo A 2009 Nucl. Instrum. Methods $600560-567$

[11] Gubser D U, Wolf S A and Cox J E 1979 Rev. Scient. Instrum. 50 751-756

[12] Dubbers D 1986 Nucl. Instrum. Methods 243511 - 517

[13] Sumner T J, Pendlebury J M and Smith K F 1987 J. Phys. D 201095

[14] Burt E A and Ekstrom C R 2002 Rev. Scient. Instrum. 73 2699-2704

[15] Wood B and Pendry J B 2007 J. Phys. Condens. Matter 19076208

[16] Navau C, Chen D X, Sanchez A and Del-Valle N 2009 Appl. Phys. Lett. 94242501

[17] Pendry J B, Schurig D and Smith D R 2006 Science 312 $1780-1782$

[18] Narayana S and Sato Y 2012 Adv. Mater. 24 71-74

[19] Magnus F, Wood B, Moore J, Morrison K, Perkins G, Fyson J, Wiltshire M C K, Caplin D, Cohen L F and Pendry J B 2008 Nat. Mater. 7 295-297

[20] Gömöry F, Solovyov M, Šouc J, Navau C, Prat-Camps J and Sanchez A 2012 Science 335 1466-1468

[21] Mansfield E H 1953 Quart. J. Mech. Appl. Math. 6 370-378

[22] Milgrom M and Shtrikman S 1989 J. Appl. Phys. 663429 3436

[23] Milton G W 2002 The Theory of Composites (Cambridge University Press, Cambridge, England)

[24] Zhou X and Hu G 2006 Phys. Rev. E 74026607

[25] Liu L P 2010 Proc. R. Soc. A 466 3659-3677

[26] Leonhardt U 2006 Science 312 1777-1780

[27] Milton G W and Nicorovici N A P 2006 Proc. R. Soc. A 462 3027-3059

[28] Milton G W, Briane M and Willis J R 2006 New J. Phys. 8248

[29] Nicorovici N A P, Milton G W, McPhedran R C and Botten L C 2007 Opt. Express 15 6314-6323

[30] Norris A N 2008 Proc. R. Soc. A 464 2411-2434

[31] Schurig D, Mock J J, Justice B J, Cummer S A, Pendry J B, Starr A F and Smith D R 2006 Science 314 977-980

[32] Alù A and Engheta N 2005 Phys. Rev. E 72016623

[33] Alù A and Engheta N 2008 Phys. Rev. Lett. 100113901

[34] Kerker M 1975 J. Opt. Soc. Am. 65 376-379

[35] Huang Y, Feng Y and Jiang T 2007 Opt. Express 1511133 11141

[36] Qiu C W, Hu L, Xu X and Feng Y 2009 Phys. Rev. E 79 047602

[37] Popa B I and Cummer S A 2009 Phys. Rev. A 79023806

[38] Xu H, Shi X, Gao F, Sun H and Zhang B 2014 Phys. Rev. Lett. 112054301

[39] Han T, Bai X, Gao D, Thong J T L, Li B and Qiu C W 2014 Phys. Rev. Lett. 112054302

[40] Kroon L 2014 Anticonductors: Cloaks for uniform static currents 8th International Congress on Advanced Electromagnetic Materials in Microwaves and Optics (Metamaterials) pp 175-177

[41] Schittny R, Kadic M, Bückmann T and Wegener M 2014 Science 345 427-429 